\title{
Balloon guide catheters: use, reject, or randomize?
}

\author{
Mayank Goyal ${ }^{1,2}\left(\right.$ D $\cdot$ Manon Kappelhof ${ }^{3}\left[\right.$ ] Johanna M. Ospel ${ }^{1,4}\left(\mathbb{C} \cdot\right.$ Fouzi Bala $^{1}(\mathbb{C}$
}

Received: 19 April 2021 / Accepted: 24 May 2021 / Published online: 8 June 2021

(c) The Author(s), under exclusive licence to Springer-Verlag GmbH Germany, part of Springer Nature 2021

\begin{abstract}
Thrombectomy devices played an important role in the success of endovascular treatment trials over the past five years. A balloon guide catheter (BGC) is an adjunctive device used to arrest and reverse flow by inflating the balloon at its tip, which allows for flow reversal in intracranial arteries during retrieval of thrombectomy devices by applying concomitant aspiration through its lumen. Thereby, it can decrease the risk of clot fragmentation and distal embolization. Despite the numerous benchtop and clinical observational studies showing the superiority of BGC to conventional guide catheters in improving reperfusion quality and clinical outcome, its use is still low in clinical practice. The reasons behind this reluctance might be related to technical and cost issues. Therefore, high level evidence data, i.e., a randomized clinical trial, are needed to increase its implementation in thrombectomy procedures. Nonetheless, several obstacles and challenges can prevent the completion of such a trial, and efforts are needed to overcome them. We provide an overview of the current opinions about BGCs in the neurointerventional community and discuss the feasibility and challenges of a possible randomized trial to answer the question "should a BGC be routinely used in endovascular treatment of acute ischemic stroke?"
\end{abstract}

Keywords Balloon guide catheter $\cdot$ Endovascular treatment $\cdot$ Randomized controlled trial

Observational evidence suggests improved results of endovascular stroke treatment (EVT) when balloon guide catheters (BGCs) are used [1]. Multiple in vitro and animal studies show that BGC use reduces distal emboli in case of clot fragmentation compared to conventional guide catheters [2]. In most clinical studies, BGCs were associated with improved reperfusion quality, greater first pass effect, shorter procedure duration, and better clinical outcomes compared to conventional guide catheters [3-8]. Only few studies, in which combined stent-retriever and aspiration is used as a first-line approach, showed no improved outcomes with BGC use [9]. Current American and European guidelines

Mayank Goyal

mgoyal@ucalgary.ca

1 Department of Clinical Neurosciences, University of Calgary, Calgary, Canada

2 Department of Diagnostic Imaging, University of Calgary, Calgary, Canada

3 Radiology and Nuclear Medicine, Amsterdam UMC, University of Amsterdam, Amsterdam, the Netherlands

4 Division of Neuroradiology, Clinic of Radiology and Nuclear Medicine, University Hospital Basel, University of Basel, Basil, Switzerland state that "the use of a BGC [...] may be beneficial" [10] and that "any EVT procedure should be performed preferably in conjunction with a proximal BGC" [11].

Nevertheless, many interventionists report that they do not routinely use BGCs. Recent studies showed that only 1 out of 4 neurointerventionalists routinely use a BGC when performing EVT [12] and that BGC use is particularly uncommon in low-volume centers [13]. What are the most common reasons for this reluctance, despite the considerably encouraging evidence available so far? Table 1 summarizes arguments against BGC use that we commonly encounter in discussions with colleagues.

If BGCs are really able to improve EVT outcomes, as suggested by the available observational data, would they be implemented in clinical practice? It seems that observational data are not enough to change current practice patterns high level evidence from a randomized trial may be needed. In theory, now would be a good time for such a trial: there seems to be clinical equipoise and sufficient momentum, since EVT indications are constantly expanding. But such a trial would face several important challenges:

1) Which thrombectomy techniques and devices should be allowed? 
Table 1 Commonly used arguments against BGC use and possible counterarguments

Arguments against BGC
BGCs are rigid and their trackability is poor. This complicates proper
BGC navigation and placement, particularly in tortuous vessels
BGCs require large femoral sheaths, which increase the risk of
iatrogenic carotid dissections and groin complications compared to
conventional catheters, especially when IV thrombolysis is used
BGCs may increase the risk of vasospasm. Maybe, interrupting blood
flow to the ischemic hemisphere during balloon inflation will lead to
further infarction

The preparation and navigation of the BGC increases procedure time

Some large bore aspiration catheters are not compatible with the currently available BGCs

BGCs may not be necessary in M1 or distal occlusions since aspiration catheters can be used to prevent embolization. Antegrade flow through the circle of Willis may limit flow reversal by BGCs

BGCs cannot be used with radial access

BGCs are expensive
Possible counterarguments

Newer BGCs are much more flexible than the older models $[14,15]$ and will likely continue this improvement

Clinically significant groin complications with BGCs are rare $(<1 \%$ even when $\geq 8$ Fr sheaths are used) [16]. Most studies did not show any difference in carotid or groin complications, including carotid dissections, with and without BGCs $[4,7]$

So far, no studies have suggested an increased risk of either infarct progression or vasospasm (though assessment is not standardized) with BGC use [3-8]. Any risk of complications should weigh up to the benefits of BGCs

Most studies reported significantly shorter (!) procedure times with BGC usage $[1,4,7,17]$, possibly owing to its high efficacy in achieving recanalization on first pass. Delays can be avoided by preparing the BGC during the waiting period after stent deployment or after turning on the aspiration pump if contact aspiration is used

Endovascular technology is rapidly evolving; new BGCs that are compatible with the largest available aspiration catheters will be available in the near future [18]

The benefit of a BGC is not so much in aspirating the clot itself, but in preventing distal embolization of clot fragments during the retrieval process, as larger clots, once they get sucked into the tip of an aspiration catheter, block the tip of the aspiration catheter. If clot fragmentation occurs in such a situation, flow arrest and reversal with a BGC in addition to distal aspiration might be beneficial as it prevents distal embolization of clot fragments [19]

New low-profile BGCs were tested successfully on the radial artery and will probably become available soon [20]. In addition, benefit of radial access over femoral access in EVT is not proven: will its benefit be larger than the benefit of BGCs? [21]

Compared with the overall cost of an EVT procedure, the costs of an additional BGC are rather low. Furthermore, in the USA, the net monetary benefit per patient per $1 \%$ increase in complete reperfusion is on average $\$ 17,000$ [22], although this number differs among countries. When translating the angiographic outcomes reported in previous studies into cost savings, the benefit of BGC probably outweighs the initial expenses by far. Generic, less expensive BGCs may also become available soon

Abbreviations: $B G C$ balloon guide catheter, $E V T$ endovascular treatment

The three broad categories of EVT techniques are primary stent-retriever, primary aspiration, and primary combined approaches (stent-retriever with distal aspiration). In order to minimize the risk of bias related to differences in thrombectomy techniques in the control and intervention arms of a hypothetical BGC trial, one would have to ensure equal and balanced distribution of the three techniques in the BGC arm and the control arm, for example, through minimization algorithms that are built in the randomization scheme.

2) What should the control arm consist of?

The most straightforward control arm to compare EVT with BGC would be EVT with a conventional guide catheter. Alternatively, a BGC could be mandated in both arms, with balloon inflation in the intervention arm only. However, this could put the control arm at a disadvantage, as there could be additional perceived difficulties associated with BGC use, such as more difficult access to the target vessel, or an increased risk of vessel dissection and vasospasm.

3) What should the primary outcome of the trial be?

While the ultimate goal is clearly to improve patients' clinical outcomes, a randomized BGC trial will unlikely show a statistically significant improvement in clinical outcomes, given the small differences in 90-day mRS in previous observational studies comparing EVT with BGC to without BGC. The sample size needed may simply be too large and the associated costs too high [7-9]. It therefore seems reasonable to use a surrogate angiographic outcome, such as successful reperfusion (eTICI 2b/3), near-complete reperfusion (eTICI 2c/3), and/or first pass effect, as primary outcome [23]. While this 
would obviate the need for long-term clinical follow-up and thereby substantially reduce trial costs, one question remains: are there reliable data on how technical success (reperfusion rate) translates into clinical outcome? A recent meta-analysis found an $11 \%$ increase in good outcome (mRS 0-2) and a 17\% increase in excellent outcome (mRS $0-1)$ with every $10 \%$ increase in TICI $2 b-3$ rates [24], which seems overoptimistic and suggests substantial bias in the available literature, begging the question of whether angiographic outcomes should be used as a surrogate for clinical outcomes in the first place. Furthermore, there is no consensus on which magnitude of improvement in angiographic outcomes is needed to change clinical practice (see discussion below).

4) What is the minimally clinical important difference (MCID)?

If a BGC trial would use an angiographic outcome (e.g., first pass eTICI $2 c / 3$ ), what should the difference in first pass eTICI $2 \mathrm{c} / 3$ rates be in order to be considered clinically meaningful? In other words: what is the minimally clinically important difference (MCID), i.e., the effect that an intervention (use of BGC in our case) should have to be worthwhile, according to physicians, patients, or both? [25] Determining the MCID is a prerequisite for determining an adequate sample size, and with that an adequate trial. Determining the MCID is not straightforward: it may vary between cultures and healthcare systems and depends on the costs and risks of the intervention, which may change over time as technology evolves. Stroke experts suggested an MCID for non-inferiority of new thrombectomy devices on reperfusion of 3.1 to $5 \%$ [26], which seems like a reasonable estimate if the goal is to get a new device approved. However, the MCID that would cause operators to change their personal EVT technique and broadly adopt BGC is likely higher. A recent meta-analysis reported a difference of $15 \%$ in first-pass reperfusion and $12 \%$ in successful final reperfusion (eTICI $2 b / 3$ ) with BGC [1], suggesting that an MCID in the range of $10-15 \%$ for successful reperfusion could be a reasonable choice. But whether this number truly is a good estimate, and whether and how it translates into improvements in clinical outcomes remains to be seen.

5) What should the secondary and safety outcomes of the trial be?

If an angiographic primary outcome is chosen, functional 90-day outcome should be included as a secondary outcome measure. Relevant other secondary outcomes would be the total procedure time, intracranial access rate, groin puncture to intracranial access time, and short-term neurological status changes (National Institutes of Health Stroke Scale).
Importantly, a BGC trial should address the raised concerns about procedural complications but should also include safety outcomes reflecting the possible beneficial effects of BGCs on complications like thrombus fragmentation and embolization. As such, safety outcomes should include (symptomatic) intracranial hemorrhage rates, mortality, infarct progression, and procedural complications like vasospasm, carotid dissection, embolization to new or downstream territories, groin hematomas, or pseudoaneurysms. Intracranial hemorrhage should be assessed using established methods like the Heidelberg Bleeding Classification [27], and infarct progression should be clearly defined (e.g., neurological deterioration not explained by intracranial hemorrhage). Crucially, procedural complications should be adjudicated based on angiography imaging by an independent core laboratory of interventionists not involved in patient management, using clear definitions for target downstream territory for assessment of embolization to new/downstream territories [28]. Previous studies showed a lower rate of angiographic safety concerns in BGC including distal embolization and emboli to new territory and no difference in procedural technical complications (dissection, perforation, and groin complication) compared to conventional guide catheters [3-8, 16]. The use of BGC was associated with a lower rate of mortality in several studies $[1,3,9]$, with no differences in symptomatic intracranial hemorrhage.

6) Which centers/operators should participate?

Operator and center experience with BGC use may play an important role in device results and study protocol implementation. Basic, pre-defined criteria for centers and operators to participate in the trial could benefit study execution and data quality. For example, a minimum number of procedures performed with BGCs could be defined, in addition to being familiar with the manufacturer's training and instructions.

7) Which patients should be included?

The most pragmatic approach would be to include all patients eligible for EVT with an anterior circulation occlusion, following the current guidelines. This would allow for generalization of the study results and increase enrolment speed. That being said, BGC efficacy may vary with occlusion location, and therefore subgroup analyses should be pre-specified in the trial design to be able to detect possible heterogeneity of treatment effect over occlusion locations.

8) How should changes in treatment technology be accounted for?

BGCs are constantly improved, and new devices are developed at a rapid pace. Substantial technological advances may be made over the course of a trial, which may change interventionalist's willingness to use 
BGCs from the perspective of offering the best possible patient care. For instance, aspiration catheters that can completely ingest the clot, rather than holding the clot at their tip when thrombus size exceeds catheter diameter, may potentially obviate the need for proximal flow arrest and flow reversal. Furthermore, adoption of radial access as standard access for acute stroke interventions may decrease BGC usage. When that happens, we will have to re-evaluate the added value of BGCs. Therefore, a trial with a relatively short enrolment period would be needed to prove BGC efficacy.

9) How can such a trial be funded?

Randomized trials are expensive. The likelihood of a BGC trial being funded by traditional peer-reviewed granting agencies such as the National Institutes of Health or similar agencies seem very low, as the question will likely be perceived to be a "niche topic" or too sub-specialized. Additionally, most of the previous major EVT trials were funded (partially) by industry, and as such, public funding agencies may feel that a BGC trial should be funded by industry as well. Indeed, industry partners would probably be willing to sponsor such a trial if they thought that the trial had a high chance to show results that increase BGC use - though these expectations should not translate into a (suspected) risk of bias, jeopardizing credibility of the trial results. On the other hand, industry partners may perceive an expected improvement in successful reperfusion of $15 \%$ as too ambitious.

In summary, substantial observational pre-clinical and clinical data point towards a beneficial effect of BGCs, but only a minority of neurointerventionalists routinely uses BGCs. The reasons for this discrepancy may include concerns about the efficacy, safety, trackability, and cost-effectiveness of BGCs. To date, no large-scale high-evidencelevel data are available to support or refute these concerns. A randomized trial could provide an answer to the question "should a BGC be used during EVT?" and would be feasible, though it would face several challenges. The stroke community should consider:

a) Do we have the collective will to conduct a randomized trial?

b) Can the trial be completed within a reasonable time frame and using a reasonable budget?

c) What should the primary outcome be: should it be an angiographic or a clinical one?

d) What is a realistic and acceptable MCID?

e) How can such a trial be funded?

Despite these challenges, we think the only way to ensure appropriate broad adoption of BGCs is through welldesigned and -conducted randomized trials.

\section{Acknowledgements None}

Funding Not applicable.

\section{Compliance with ethical standards}

Conflict of interest Mayank Goyal is a consultant for Medtronic, Stryker, Microvention, Mentice, GE Healthcare, and holds a licensing agreement with GE Healthcare regarding systems of acute stroke diagnosis. Fouzi Bala is supported by la Société Française de Radiologie et la Société Française de Neuroradiologie. The remaining authors have nothing to disclose.

Ethical approval Not applicable since this is a review article and no patient data are reported in this manuscript.

Informed consent Not applicable since this is a review article and no patient data are reported in this manuscript.

\section{References}

1. Brinjikji W, Starke RM, Murad MH et al (2018) Impact of balloon guide catheter on technical and clinical outcomes: a systematic review and meta-analysis. J Neurointerv Surg 10:335-339. https:// doi.org/10.1136/neurintsurg-2017-013179

2. Chueh J-Y, Kang D-H, Kim BM, Gounis MJ (2020) Role of balloon guide catheter in modern endovascular thrombectomy. J Korean Neurosurg Soc 63:14-25. https://doi.org/10.3340/jkns. 2019.0114

3. Nguyen TN, Castonguay AC, Nogueira RG et al (2019) Effect of balloon guide catheter on clinical outcomes and reperfusion in Trevo thrombectomy. J Neurointerv Surg 11:861-865. https://doi. org/10.1136/neurintsurg-2018-014452

4. Velasco A, Buerke B, Stracke CP et al (2016) Comparison of a balloon guide catheter and a non-balloon guide catheter for mechanical thrombectomy. Radiology 280:169-176. https://doi. org/10.1148/radiol.2015150575

5. Zaidat OO, Mueller-Kronast NH, Hassan AE et al (2019) Impact of balloon guide catheter use on clinical and angiographic outcomes in the STRATIS stroke thrombectomy registry. Stroke 50:697-704. https://doi.org/10.1161/STROKEAHA.118.021126

6. Kang D-H, Kim BM, Heo JH et al (2018) Effect of balloon guide catheter utilization on contact aspiration thrombectomy. J Neurosurg 131:1494-1500. https://doi.org/10.3171/2018.6.JNS181045

7. Baek J-H, Kim BM, Kang D-H et al (2019) Balloon guide catheter is beneficial in endovascular treatment regardless of mechanical recanalization modality. Stroke 50:1490-1496. https://doi.org/10. 1161/STROKEAHA.118.024723

8. Goldhoorn R-JB, Duijsters N, Majoie CBLM et al (2019) Balloon guide catheter in endovascular treatment for acute ischemic stroke: results from the MR CLEAN registry. J Vasc Interv Radiol 30:1759-1764.e6. https://doi.org/10.1016/j.jvir.2019.05.032

9. Bourcier R, Marnat G, Labreuche J et al (2020) Balloon guide catheter is not superior to conventional guide catheter when stent retriever and contact aspiration are combined for Stroke treatment. Neurosurgery 88:E83-E90. https://doi.org/10.1093/neuros/nyaa3 15

10. Powers WJ, Rabinstein AA, Teri A et al (2019) Guidelines for the early management of patients with acute ischemic stroke: 2019 update to the 2018 guidelines for the early management of acute 
ischemic stroke: a guideline for healthcare professionals from the American Heart Association/American Stroke Association. Stroke 50:e344-e418. https://doi.org/10.1161/STR.0000000000000211

11. Turc G, Bhogal P, Fischer U et al (2019) European Stroke Organisation (ESO)- European Society for Minimally Invasive Neurological Therapy (ESMINT) guidelines on mechanical thrombectomy in acute ischemic stroke. J Neurointerv Surg 11:535-538. https:// doi.org/10.1136/neurintsurg-2018-014568

12. Mehta T, Male S, Quinn C et al (2019) Institutional and provider variations for mechanical thrombectomy in the treatment of acute ischemic stroke: a survey analysis. J Neurointerv Surg 11:884-890. https://doi.org/10.1136/neurintsurg-2018-014614

13. Nogueira RG, Haussen DC, Alicia C et al (2019) Site experience and outcomes in the Trevo acute ischemic stroke (TRACK) multicenter registry. Stroke 50:2455-2460. https://doi.org/10.1161/ STROKEAHA.118.024639

14. Yi HJ, Sung JH, Lee MH, Lee DH (2019) Experience of the New FlowGate2 Device as a balloon guide Catheter For Ischemic Stroke Intervention. World Neurosurg 126:e736-e742. https:// doi.org/10.1016/j.wneu.2019.02.140

15. Walrus : Q’Apel Medical Inc. https://qapelmedical.com/walrus/. Accessed 24 Mar 2021

16. Shah VA, Martin CO, Hawkins AM et al (2016) Groin complications in endovascular mechanical thrombectomy for acute ischemic stroke: a 10-year single center experience. J Neurointerv Surg 8:568-570. https://doi.org/10.1136/neurintsurg-2015-011763

17. Nguyen TN, Malisch T, Castonguay AC et al (2014) Balloon guide catheter improves revascularization and clinical outcomes with the Solitaire device: analysis of the North American Solitaire Acute Stroke Registry. Stroke 45:141-145. https://doi.org/10.1161/ STROKEAHA.113.002407

18. K193607.pdf Accessed March 24, 2021. https://www.accessdata. fda.gov/cdrh_docs/pdf19/K193607.pdf

19. Ospel JM, Volny O, Jayaraman $\mathrm{M}$ et al (2019) Optimizing fast first pass complete reperfusion in acute ischemic stroke - the BADDASS approach (BAlloon guiDe with large bore Distal Access catheter with dual aspiration with Stent-retriever as Standard approach). Expert Rev Med Devices 16:955-963. https://doi.org/ 10.1080/17434440.2019.1684263

20. Chen SH, Snelling BM, Sur S et al (2019) Transradial versus transfemoral access for anterior circulation mechanical thrombectomy: comparison of technical and clinical outcomes. Journal of
NeuroInterventional Surgery 11:874-878. https://doi.org/10.1136/ neurintsurg-2018-014485

21. Siddiqui AH, Waqas M, Neumaier J et al (2021) Radial first or patient first: a case series and meta-analysis of transradial versus transfemoral access for acute ischemic stroke intervention. J Neurointerv Surg. https://doi.org/10.1136/neurintsurg-2020-017225

22. Kunz WG, Almekhlafi MA, Menon BK et al (2020) Public health and cost benefits of successful reperfusion after thrombectomy for stroke. Stroke 51:899-907. https://doi.org/10.1161/STROK EAHA.119.027874

23. Zaidat OO, Castonguay AC, Linfante I et al (2018) First pass effect: a new measure for stroke thrombectomy devices. Stroke 49:660-666. https://doi.org/10.1161/STROKEAHA.117.020315

24. Manning NW, Warne CD, Meyers PM (2018) Reperfusion and clinical outcomes in acute ischemic stroke: systematic review and meta-analysis of the stent-retriever-based, early window endovascular stroke trials. Front Neurol 9:301. https://doi.org/10.3389/ fneur.2018.00301

25. Jaeschke R, Singer J, Guyatt GH (1989) Measurement of health status. Ascertaining the minimal clinically important difference. Control Clin Trials 10:407-415. https://doi.org/10.1016/01972456(89)90005-6

26. Lin C-J, Saver JL (2020) The minimal clinically important difference for achievement of substantial reperfusion with endovascular thrombectomy devices in acute ischemic stroke treatment. Front Neurol 11:524220. https://doi.org/10.3389/fneur.2020.524220

27. von Kummer R, Broderick JP, Campbell BCV et al (2015) The Heidelberg Bleeding Classification: classification of bleeding events after ischemic stroke and reperfusion therapy. Stroke 46:2981-2986. https://doi.org/10.1161/STROKEAHA.115. 010049

28. Zaidat OO, Yoo AJ, Khatri P et al (2013) Recommendations on angiographic revascularization grading standards for acute ischemic stroke. Stroke 44:2650-2663. https://doi.org/10.1161/ STROKEAHA.113.001972

Publisher's Note Springer Nature remains neutral with regard to jurisdictional claims in published maps and institutional affiliations. 